\title{
DDDAS for Fire and Agent Evacuation Modeling of the Rhode Island Nightclub Fire
}

\author{
Alok Chaturvedi ${ }^{1}$, Angela Mellema ${ }^{1}$, Sergei Filatyev ${ }^{2}$, and Jay Gore ${ }^{2}$ \\ ${ }^{1}$ Purdue Homeland Security Institute, Purdue University, West Lafayette, IN 47906 \\ \{alok, amellema\}@purdue. edu \\ ${ }^{2}$ School of Mechanical Engineering, Purdue University, West Lafayette, IN 47906 \\ \{sfilatye, gore\}@purdue.edu
}

\begin{abstract}
A Dynamic Data Driven Application System (DDDAS) was created to study interaction between fire and agent models during a fire evacuation. The analysis from that research can be used to validate proposed ideas in evacuation and building designs to ensure safety of buildings given various agent behaviors. Two separate models were used to simulate the components of the emergency situation: fire and agent. The independent models were able to run using data computed by the other interacting models, allowing careful examination of real-time interactions in a situation. Through study of the interactions, a better understanding is gained of how individual variables such as exit position and width affect the evacuation process and escape rate in the given scenario. Exits can be relocated and changed to quickly assess the effect on the model. The results can be used for improving building design and regulations as well as training first responders.
\end{abstract}

\section{Introduction}

In the event of an emergency situation, skilled planning and response preparation can dramatically affect the results of the scenario. In order to prepare a response for such an event it is important to study each important aspect of the model in order to understand how changes in the environment affect the outcome of the emergency situation. The scenario presented in this paper is modeled after The Station nightclub fire of West Warwick, Rhode Island which took place on February 20, 2003. In the case of a fire such as that described in the present paper the important aspects of the model are fire propagation and human evacuation. The fire and agent simulations are run simultaneously. By creating a virtual environment where simulations are run simultaneously the factors that affect each model can be monitored and measured to learn how to better prepare for and respond to an emergency situation.

Realistic models describing fire propagation and human behavior were needed for this comprehensive simulation. Currently, models exist which simulate fire and human behavior during evacuation, but those models have yet to be integrated to simulate real-time events and decisions [1]. As a result of studying key events of an emergency scenario, decisions can be made about emergency response and building planning prior to the event. 


\section{Models}

The present scenario was modeled after The Station nightclub fire. The National Institute of Standards in Technology has completed a thorough investigation and computer simulation of that fire [2]. The research completed by NIST was used to validate the current project, and as a foundation for continued research. Figure 1 shows the basic floor plan of the nightclub.

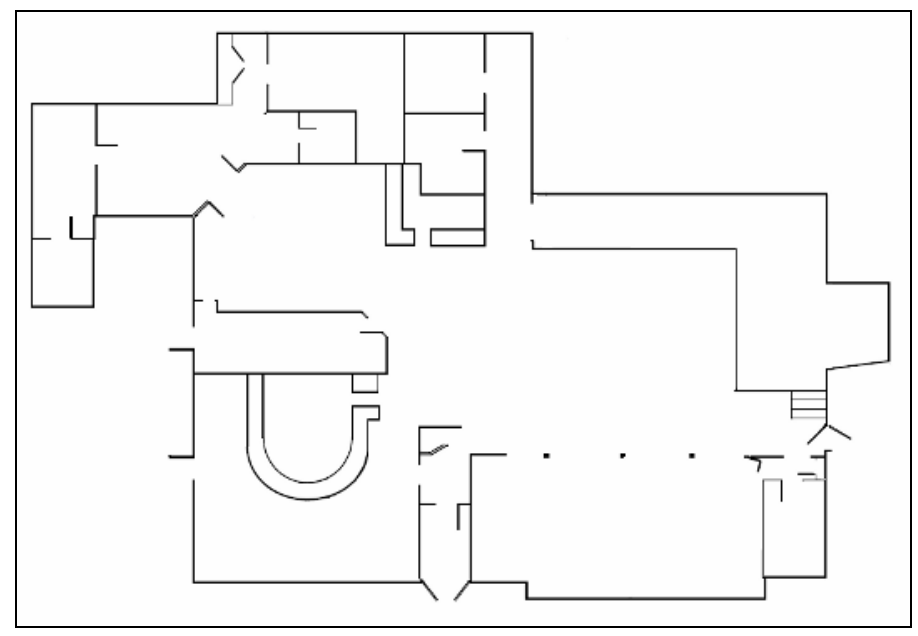

Fig. 1. Basic floor plan of The Station nightclub. The exit at the bottom of the picture is the main exit. The exit on the right was the exit closest to the stage.

There are four exits in the building: the main exit (bottom of Fig. 1), the stage exit (right of Fig. 1), the kitchen exit (top left of Fig. 1), and the bar exit (bottom left of Fig.1). The fire began on the stage, very close to the stage exit, and began as a result of a pyrotechnics display igniting polyurethane foam that was lining the walls of the stage and dance floor. The main exit was the exit used by the majority of the people inside of the building, due to a familiarity with that exit.

\subsection{Fire}

Fire was simulated using the Fire Dynamics Simulator, FDS4, developed by NIST [3]. FDS4 is a large-eddy simulation (LES) turbulent code which employs the mixture fraction model. Simulations run using FDS4 have been successfully used to describe different processes from fundamental combustion studies of plumes to the replication of the 2001 fire in the World Trade Center [4]. FDS provides time-resolved temperature, carbon monoxide, carbon dioxide and soot distribution throughout the building for the duration of the fire. These calculations show how the fire and smoke propagate through the building and the results were used in the agent evacuation model.

After the fire occurred at The Station nightclub, NIST used FDS to extensively simulate the progression of the fire [2]. Most of the materials that were present in the building at the time of the fire were tested and the combustive properties of those 
materials were used within the FDS simulation. The input file used by NIST was also used for the fire simulation in the current paper [5].

\subsection{Agent}

The agent model was designed to simulate agent behaviors during evacuation. In the case of a fire in a densely populated nightclub, crowd effects were considered and implemented.

Agent movements within the environment were simulated using a generalized force model described by Helbing, Farkas \& Vicsek [6]. Agents exist in an environment in which socio-psychological and physical forces are exerted upon each agent influencing their behavior. Each agent $i$ is pulled toward their goal position, the exits, in a certain direction $\mathrm{e}_{i}^{0}$ at certain desired speeds $v_{i}^{0}$ according to their mass $m$, and this attraction determines their evacuation path. Each agent adapts their actual velocity $\mathrm{v}_{i}$ to a certain time $\tau_{i}$. Interaction forces of other agents $j$ and walls $W$ are represented by $\mathrm{f}_{i j}$ and $\mathrm{f}_{i W}$, and also control the velocity of each agent. The following equation was used to calculate acceleration.

$$
m_{i} \frac{\mathrm{dv}_{i}}{\mathrm{~d} t}=m_{i} \frac{v_{i}^{0}(t) \mathrm{e}_{i}^{0}(t)-\mathrm{v}_{i}(t)}{\tau_{i}}+\sum_{j(\neq i)} \mathrm{f}_{i j}+\sum_{W} \mathrm{f}_{i W}
$$

The forces exerted upon the agents from objects such as walls and agents also can affect the agent's position. Two agents $i$ and $j$ tend to stay away from each other as they are moving in a given direction based on a repulsive force $A_{i} \exp \left[\left(r_{i j}-d_{i j}\right) / B_{i}\right] \mathrm{n}_{i j}$, where $A_{i}$ and $B_{i}$ are constants.

$$
\mathrm{f}_{i j}=\left\{A_{i} \exp \left[\left(r_{i j}-d_{i j}\right) / B_{i}\right]\right\} \mathrm{n}_{i j}
$$

Lastly, the agents interact with walls and other obstructions throughout the environment. $d_{i W}$ represents the distance to the wall $W, \mathrm{n}_{i W}$ represents the direction perpendicular to it. The final equation denoting the interaction between agents and other obstructions is given by:

$$
\mathrm{f}_{i W}=\left\{A_{i} \exp \left[\left(r_{i}-d_{i W}\right) / B_{i}\right]\right\} \mathrm{n}_{i W}
$$

Agents choose to evacuate out of a particular exit based on the agent's familiarity with the building. Different agents can be aware of certain exits based upon where they are located and any previous knowledge of the building. This is represented in the simulation by giving each agent a varying level of knowledge of the building's exits. As the conditions in the building worsen the agent may chose to change their route based upon their personal safety. For example, as many agents are attempting to leave out of one exit the exit may become blocked and, as the conditions in the building worsen, agents may attempt to find another exit.

Work is currently being done to allow the fire to affect the health of each agent based on ISO standard 13571 [7]. Fractional Effective Doses (FED) for heat exposure 
and gas concentration for each time $t$ will be calculated and used to lower the health of the agents appropriately.

\section{Model Interaction}

Agents can take actions such as breaking windows and opening doors. These actions have an effect on how the fire moves throughout the model.

Currently agent's actions can only fuel fire by opening windows and doors, agents cannot take action against the fire. This functionality is in development. Since no significant fire fighting measures were taken in the current scenario, this implementation was not necessary.

\section{Results}

Three cases of the scenario were computed for the fire evacuation. The first two cases were similar to the cases used in the research conducted by NIST [2]. The first case was calculated allowing all agents to leave out of each exit and each agent chose the exit that was closest to them. The exit times of each agent were monitored, showing the best evacuation time in optimal building conditions, 226 seconds.

As the first case does not accurately represent the conditions in the building, the second case was designed to reflect the conditions of The Station nightclub more closely. In this case, the exit that is closest to the fire's point of origin is considered impassible at the time that the fire consumes the area, when time is $t=30$ seconds. The second exit that became impassible was the main exit, which became blocked due to crowd crush. This happened at $\mathrm{t}=90$ seconds. When these two exits were blocked at their respective times, the time of evacuation grew to 346 seconds. Figure 2 shows the

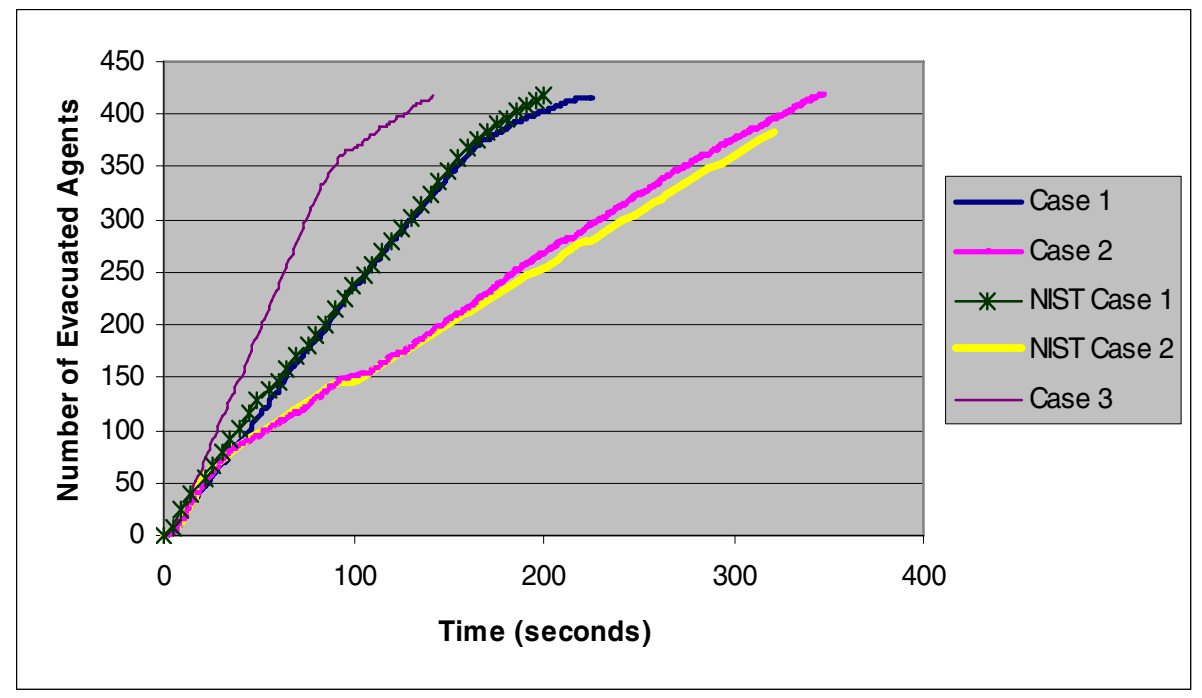

Fig. 2. The rates of evacuation for the first two cases of the scenario plotted with the rates of evacuation in the research completed by NIST 
rates of evacuation during the two cases. The slope of the data line for Case 2 in figure 2 decreases at the time of the two exit closings, showing the affect of the closings on the overall evacuation rate.

The first two cases were replications of the NIST research, and were computed to benchmark the current system. These two cases corresponded very closely with the research completed by NIST. The blocking of the two exits in the second case affected the rate of evacuation similarly and the corresponding cases took roughly the same amount of time to evacuate.

A final case, Case 3 of Figure 1, was computed using the DDDAS to calculate the effect of modifying areas of the building that impede evacuation. Figure 3 shows how the main exit area was changed to calculate Case 3.

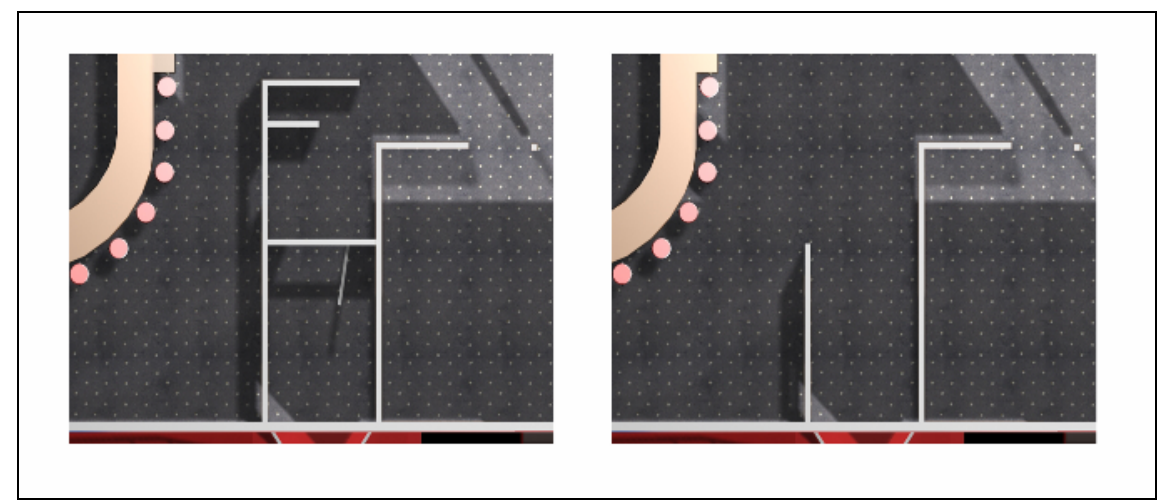

Fig. 3. The image on the left is the model used to represent the nightclub on the night of the fire. The image on the right is the modified model used to asses the affect on evacuation

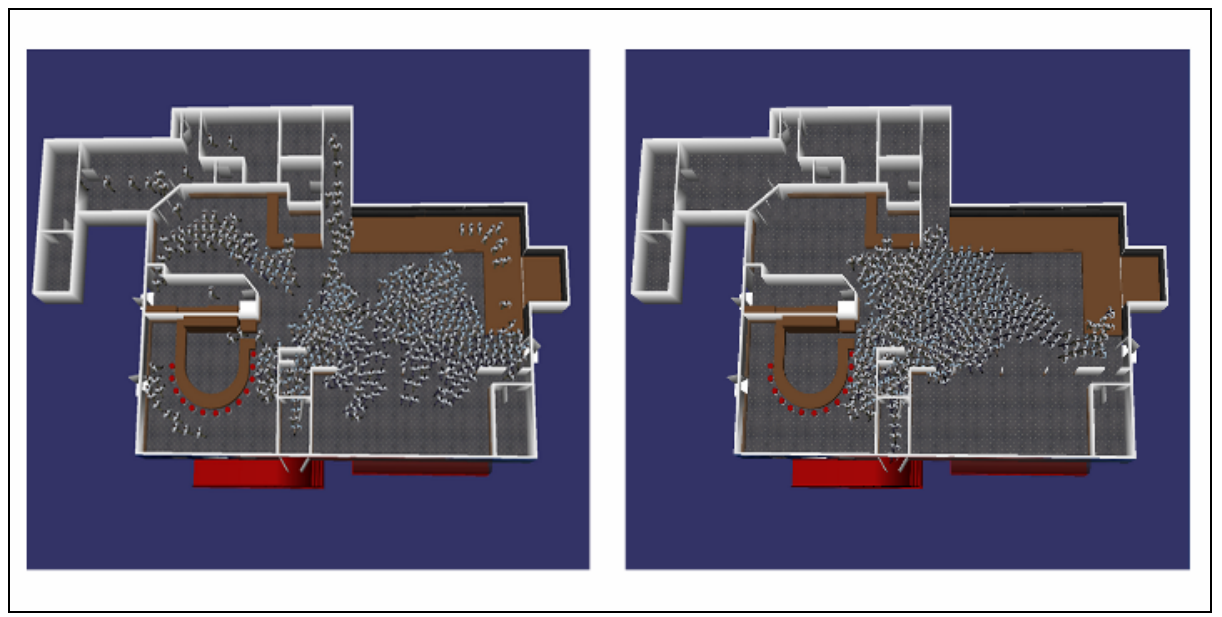

Fig. 4. The image on the left is the evacuation visualization at 5 seconds; the image on the right is the evacuation visualization at 30 seconds. The Case 1 visualization is shown 
This simple change to the geometry of the building greatly affected the amount of "crowd crush" that was experienced. The third case was a modification of the first case, in which the exits were not blocked at any time. Agents each chose the exit closest to them. In comparison to Case 1, all agents that were closest to the main exit were able to evacuate out of the main exit even faster than the agents were able to exit out of the stage exit. This is a considerable improvement over the first case. The total time of evacuation for the third case was 143 seconds, as opposed to the time of evacuation for the third case, 226 seconds.

The visualization of the models is an important part of the DDDAS. It allows us to see clearly effects of potential problems, e.g. exit blockage, and to help finding solutions to them. Figure 4 is the visualization at $t=5$ and $t=30$ seconds of Case 1 .

The fire spreads rapidly throughout the building. The visualization shown in Figure 5 shows the rapid spread of fire throughout the main room of The Station nightclub.
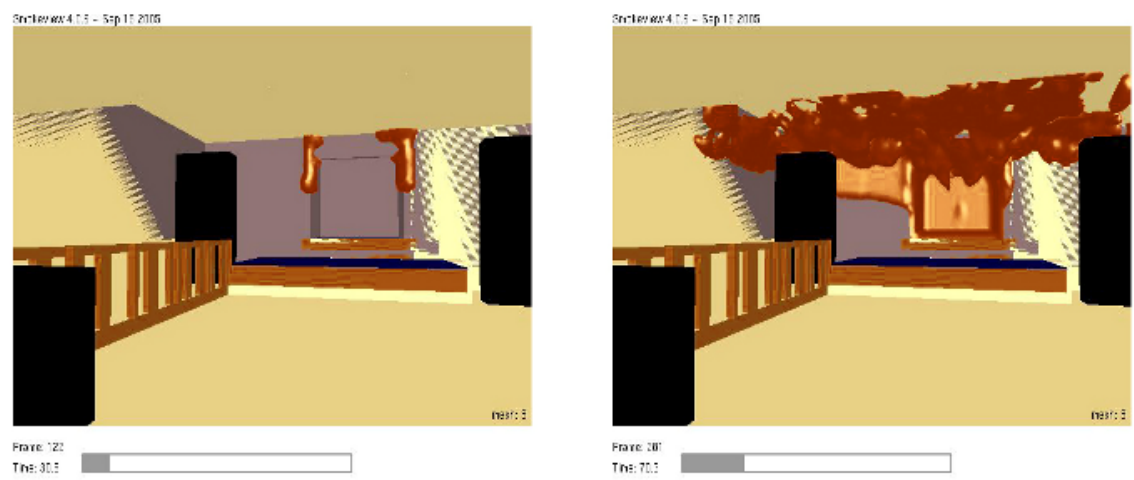

Fig. 5. Fire visualization. The image on the left is the visualization at 30 seconds; the image on the right is the visualization at 70 seconds.

Smoke is not shown in the above figure so that the flame position can be clearly seen. Just 70 seconds after ignition the stage area is consumed by flames and the main room is filled with smoke.

\section{Conclusions}

The Dynamic Data Driven Shared Reality System was created to study the events of The Station nightclub fire of 2003. Two scenarios were run to closely match the research completed by NIST shortly after the 2003 disaster. The results of those two scenarios gave similar results to draw the same conclusions as the NIST paper.

That research was then expanded upon to include the effect of fire upon the agents during evacuation. A third scenario was run testing how changes to the geometry can affect the total evacuation time as well as changes to the evacuation process. Simulations allow effects of these changes to be instantly visible and make it possible to set up many configurations of the building layout. Building planning, design and modification can then be considered more intelligently and also justified with data supporting 
certain decisions. Real-time and near-real-time visualizations also assist with data collection and viewing the results of such changes.

\section{References}

1. Foong, C., Armstrong, B., Dilley, D., Grahn, J., Krull, K., Chaturvedi, A., Gore, J., Filatyev, S.: Towards Enabling a Distributed and Scalable Society of Simulations. 2005 Spring Simulation Multiconference (SpringSim '05). (2005)

2. Grosshandler, W., Bryner, N., Madrzykowski, D., Kuntz, K.: Report of the Technical Investigation of The Station Nightclub Fire. NIST NCSTAR 2, Vol. 1 (2005)

3. McGrattan, K., Forney, G.: Fire Dynamics Simulator (Version 4). Users Guide. NIST Special Publication 1019. 2004 <http://fire.nist.gov/fds>

4. Prasad, K., Baum, H.: Coupled Fire Dynamics and Thermal Response of Complex Building Structures. Proc. Combust. Inst. 30 (2005) 2255-2262

5. Grosshandler, W., Bryner, N., Madrzykowski, D., Kuntz, K.: Report of the Technical Investigation of The Station Nightclub Fire: Appendices. NIST NCSTAR 2, Vol. 2 (2005)

6. Helbing, D., Farkas, I., Vicsek, T.: Simulating Dynamical Features of Escape Panic. Nature, Vol. 407. (2000) 487-490.

7. International Organization for Standardization: Life-threatening components of fire Guidelines for the estimation of time available for escape using fire data, ISO/TS 13571:2002. (2002)

8. Purser, D.A.: Toxicity Assessment of Combustion Products, SFPE Handbook of Fire Protection Engineering, P.J. DiNenno, Ed., $2^{\text {nd }}$ Ed., National Fire Protection Association, Quincy, MA, Sec.2, (1995) 85-146 